\title{
Molecular mechanism of HIF-1-independent VEGF expression in a hepatocellular carcinoma cell line
}

\author{
SAE BYEOL CHOI ${ }^{1}$, JONG BAE PARK $^{2}$, TAE-JIN SONG ${ }^{3}$ and SANG YONG CHOI ${ }^{1}$ \\ ${ }^{1}$ Department of Surgery, Korea University College of Medicine, Korea University Guro Hospital, Guro-gu, Seoul 152-703; \\ ${ }^{2}$ Laboratory of Functional Genomics, Department of Neurosurgery, Research Institute and Hospital, National Cancer \\ Center, Ilsan-gu, Goyang, Gyeonggi 411-764; ${ }^{3}$ Department of Surgery, Korea University College of Medicine, \\ Korea University Ansan Hospital, Danwon-gu, Ansan, Kyunggi-do 425-707, Republic of Korea
}

Received February 17, 2011; Accepted April 11, 2011

DOI: $10.3892 / \mathrm{ijmm} .2011 .719$

\begin{abstract}
Hypoxia-inducible factor-1 (HIF-1) is a master transcription factor that plays a central role in the hypoxic expression of various genes. Vascular endothelial growth factor (VEGF), a known target gene of HIF-1 $\alpha$, has been shown to be induced by hypoxia through a HIF-1 $\alpha$-independent pathway. HIF-1 $\alpha$ dominant-negative lentiviral vectors were introduced to decrease the expression of HIF in Hep3B cells. Cells were incubated under normoxic or hypoxic conditions. We performed a VEGF enzyme-linked immunosorbent assay (ELISA) using cell culture supernatants, and Western blotting using cell lysates. To validate signaling via HIF-1-dependent or HIF-1-independent pathways, we treated cells with an extracellular signal-regulated kinase (ERK) kinase inhibitor, a phosphoinositide 3-kinase (PI3K) inhibitor, and transfected cells with siSP1. HIF-1 $\alpha$ protein expression was induced and the levels of VEGF increased under hypoxic conditions. Cells were transfected with siHIF-1 $\alpha$ and incubated under normoxic or hypoxic conditions. We found that a significant amount of VEGF was produced by a HIF-1-independent pathway. PI3K inhibitor treatment and siSP1 transient transfection decreased VEGF expression in siHIF- $1 \alpha$-transfected cells. Therefore, VEGF regulation in Hep3B cells is primarily controlled by the Akt/PI3K and SP1 pathways and is independent of HIF-1 under hypoxic conditions.
\end{abstract}

\section{Introduction}

Most solid tumors contain hypoxic lesions due to an imbalance in oxygen supply and consumption. Under hypoxic conditions, normal cells are unable to tolerate ischemia resulting in cell

Correspondence to: Dr Tae-Jin Song, Department of Surgery, Korea University College of Medicine, Korea University Ansan Hospital, 516 Gojan-dong, Danwon-gu, Ansan, Kyunggi-do 425-707, Republic of Korea

E-mail: tjsong@korea.ac.kr

Key words: hepatocellular carcinoma, Hep3B cell line, angiogenesis, vascular endothelial growth factor, hypoxia-inducible factor-1, SP1 death. Although hypoxia is toxic to both cancer cells and normal cells, cancer cells undergo genetic and adaptive changes that allow them to survive and even proliferate in a hypoxic environment (1). Clinical and experimental evidence suggests that hypoxic processes in cancer cells contribute to the malignant phenotype and to more aggressive tumor behavior (2). Hypoxia offers resistance to anticancer chemotherapy, as well as a predisposition to increased tumor metastases (3). Investigation of the molecular mechanisms specific to cancer cells under hypoxic conditions is important to provide a basis for anticancer therapy.

Hypoxia-inducible factor-1 (HIF-1) is a transcription factor found in mammalian cells that plays a central role in the hypoxic expression of various genes including vascular endothelial growth factor (VEGF). VEGF is one of the first isolated and the most potent angiogenic factor (4). VEGF has an important role during developmental, physiological and pathological neovascularization (5). VEGF is known to be one of the target genes of HIF-1 $\alpha$.

Hepatocellular carcinoma (HCC) is well known as a hypervascular tumor; however, it is hypovascular in its early stage and the rapid proliferation of tumor cells continuously induces local hypoxia in its advanced stage (6). Pharmacological inhibition of the HIF target, VEGF, has proven to be efficacious as a cancer treatment (7). However, research into how the specific regulation of VEGF in cancer cells is different from VEGF regulation in normal cells is important for the development of anticancer drugs that specifically act on cancer cells to reduce side effects.

It has been reported that several genes are not directly regulated by HIF-1 $\alpha$ (HIF-1 independent pathway) under hypoxic conditions (8-10). These findings suggest the existence of HIF-1 $\alpha$-independent molecular pathways that promote tumor progression under hypoxic conditions. The growth of HCC depends on stimulatory effects of assorted growth factors, which bind to tyrosine kinase receptors to activate various intracellular signaling pathways that contribute to tumor cell proliferation, tumor cell survival, migration, and metastasis under hypoxia (4). Under hypoxic conditions, the growth signaling pathways of tumor cells are activated, while in normal cells they are not. It has been reported that growth factor inhibitors, such as EGFR tyrosine kinase inhibitors, 
decrease VEGF expression via a HIF-1-independent mechanism through SP1 (11). Therefore, we hypothesized that under hypoxic conditions growth signaling pathways induce HIF-1independent VEGF expression in cancer cell proliferation. In the present study, we demonstrate that the molecular mechanism of the growth signaling pathway of VEGF expression in the Hep3B cell line is HIF-1 independent.

\section{Materials and methods}

Establishment of the siHIF-1 $\alpha$-transfected Hep3B cell line. Short hairpin small interfering RNA (siRNA) specific for HIF-1 $\alpha$ dominant-negative lentiviral vector was introduced into Hep3B cells to decrease the expression of HIF-1. To establish the lentiviral vector, a HIF expression vector for siHIF-1 $\alpha$ was manufactured using the MultiSite-Gateway system (Invitrogen, Carlsbad, CA, USA) according to the manufacturer's instructions. 293FT cells were transfected with the expression vector and packaging plasmid (pMDL, pVSV, pRSV). After $72 \mathrm{~h}$, the supernatant was collected and the Hep3B cell line was infected by the supernatant containing lentiviral particles. Transfection efficiency in Hep3B cells was measured by co-expression of green fluorescent protein (GFP).

Cell culture and exposure to hypoxia. The Hep3B cell line was maintained and cultured in RPMI-1640 media supplemented with $10 \%$ heat-inactivated fetal bovine serum, $100 \mu \mathrm{g} / \mathrm{ml}$ streptomycin, and $100 \mathrm{U} / \mathrm{ml}$ penicillin. Hep3B cells were incubated at $37^{\circ} \mathrm{C}$ in a humidified atmosphere containing $20 \% \mathrm{O}_{2}$ and $5 \% \mathrm{CO}_{2}$ in air; this is referred to as the normoxic condition. For the hypoxia experiments, cells were placed in a hypoxic chamber containing $1 \% \mathrm{O}_{2}, 5 \% \mathrm{CO}_{2}$, and $94 \% \mathrm{~N}_{2}$, and maintained at $37^{\circ} \mathrm{C}$. In brief, $2.5 \times 10^{5}$ cells/well were seeded in 6-well plates 1 day before incubation under normoxic or hypoxic conditions at a $37^{\circ} \mathrm{C}$ humidified atmosphere. After $24 \mathrm{~h}$, the tissue culture supernatant was collected from each well.

Protein extraction and Western blot analysis. For protein isolation, cells were solubilized in $100 \mu \mathrm{l}$ chilled lysis buffer containing Complete Mini Protease Inhibitor tablets (Roche Molecular Biochemicals, Mannheim, Germany), phenylmethylsulphonyl fluoride, phosphatase inhibitor, lysis buffer and distilled water. Samples were centrifuged at 12,000 x g and the supernatants were retained. The protein concentrations were determined using a Quanti-iT Protein Assay kit (Invitrogen). Each protein sample was mixed with SDS-PAGE loading buffer and boiled at $85^{\circ} \mathrm{C}$ for $2 \mathrm{~min}$.

For Western blotting, equal amounts of total protein were run in each lane of an SDS-PAGE gel. After completion of gel electrophoresis, proteins were transferred to nitrocellulose membrane (Protran, Whatman, Dassel, Germany) for $1 \mathrm{~h}$ using a blotting apparatus at $4^{\circ} \mathrm{C}$. The membrane was incubated with each specific antibody at $4^{\circ} \mathrm{C}$ for over $16 \mathrm{~h}$ followed by a secondary antibody at room temperature for $1 \mathrm{~h}$. The blots were probed with anti-HIF-1 $\alpha$ (R\&D Systems, Minneapolis, $\mathrm{MN}$ ), anti- $\beta$-actin (Santa Cruz Biotechnology, Santa Cruz, CA), anti-phospho-p44/42 MAPK (Cell Signaling Technology, Beverly, MA), at 1:1,000 dilutions. Immunoreactive proteins were visualized using the ECL Plus Western blotting detection reagent (Amersham Biosciences, Uppsala, Sweden).
Reverse transcription-polymerase chain reaction (RT-PCR). Hep3B cells were incubated under normoxic or hypoxic conditions for $24 \mathrm{~h}$. Total RNA was isolated with TRIzol (Invitrogen) following the instructions of the manufacturer. RT-PCR was performed using a commercial reverse transcription system kit (Promega, Madison, WI). RNA sample (1 $\mu \mathrm{g})$ was converted into cDNA using random primers and reverse transcriptase. Amplification by PCR was performed using Takara Taq DNA polymerase (Takara Biochemicals, Shiga, Japan) according to the manufacturer's instructions. Thermal cycling was performed for 26 cycles using the following steps: denaturing at $94^{\circ} \mathrm{C}$ for $30 \mathrm{sec}$, annealing at $51^{\circ} \mathrm{C}$ for $30 \mathrm{sec}$, and extension at $72^{\circ} \mathrm{C}$ for 90 sec. The sequences of the PCR primer pairs were: VEGF forward, 5'-GCTACTGCCATCCA ATCGAG-'3; VEGF reverse, 5'-TGCATTCACATTTGTTGT GC-'3; GAPDH forward, 5'-ACCACAGTCCATGCCATCAC-'3; and GAPDH reverse, 5'-TCCACCACCCTGTTGCTGTA-'3.

Measurement of VEGF from the tissue culture supernatant. The VEGF levels in the tissue culture supernatant collected from cells after incubation under normoxic or hypoxic conditions for $24 \mathrm{~h}$ were measured using the human VEGF enzyme linked immunosorbent assay (ELISA) kit (BioSource International, CA).

Cell treatment with PI3K and ERK kinase inhibitors. Cells $\left(2.5 \times 10^{5}\right.$ per well) were seeded in 6-well plates 1 day before treatment with the PI3K inhibitor, LY294002 (Cell Signaling Technology) or the ERK kinase inhibitor, PD98059 (Cell Signaling Technology). The cells were pretreated with $50 \mu \mathrm{M}$ inhibitor or left untreated for $1 \mathrm{~h}$ and then incubated under normoxic or hypoxic conditions for $24 \mathrm{~h}$. Cell supernatants were collected for VEGF ELISA and the proteins were extracted from the cells for analysis by Western blotting.

Transient transfection. Cells $\left(1.5 \times 10^{5}\right.$ per well) were seeded in 6 -well plates before transfection using the FuGENE 6 transfection reagent (Roche, Indianapolis, IN), according to the instructions of the manufacturer. Briefly, $6 \mu$ of FuGENE 6 reagent was diluted with serum-free medium. siRNA $(40 \mathrm{mM})$ was added to the diluted FuGENE 6 reagent, and then incubated for up to $45 \mathrm{~min}$ at room temperature. The complex formed was then added to each well and the cells were returned to the incubator. The medium was replaced with serumcontaining medium $8 \mathrm{~h}$ after transfection. After $48 \mathrm{~h}$, cells were incubated under normoxic or hypoxic conditions for $24 \mathrm{~h}$ and the supernatant was collected for measurement of VEGF by ELISA.

Statistical analysis. Data are expressed as the mean \pm SEM. Statistical calculations were performed using SPSS, version 13.0 for Windows (SPSS Inc. Chicago, IL). Comparison of the mean values was performed with the unpaired t-test. Statistical significance was accepted at $\mathrm{P}<0.05$.

\section{Results}

HIF-1 $\alpha$ and VEGF expression in siHIF-1 $\alpha$-transfected cells under normoxic or hypoxic conditions. To examine the effects of HIF-1 in Hep3B cells under hypoxic conditions, Hep3B 
A

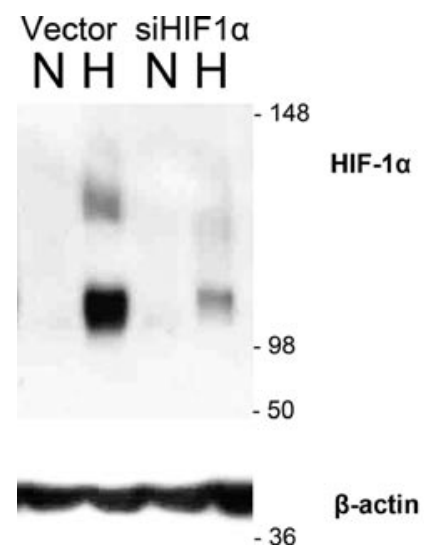

B

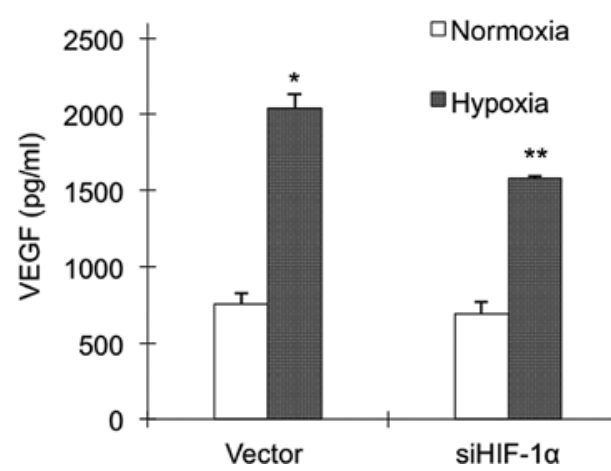

Figure 1. HIF-1 $\alpha$ and VEGF expression by siHIF-1 $\alpha$ transfected cells under normoxic or hypoxic conditions. The expression of HIF-1 $\alpha$ decreased markedly in siHIF-1 $\alpha$-transfected cells (A). VEGF expression decreased in siHIF-1 $\alpha$ transfected cells under hypoxia; however significant VEGF expression was still detectable in siHIF-1 $\alpha$-transfected cells under hypoxia (B). N, normoxia; ${ }^{*} \mathrm{p}<0.01$ vs. vector normoxia; H, hypoxia; ${ }^{* *} \mathrm{p}<0.05$ vs. vector hypoxia.

cells were transfected by vector (for control) and siHIF-1 $\alpha$ using lentiviral vectors to induce stable expression. The efficiency of the transfection was identified by GFP signals. We confirmed that almost all cells were sufficiently transfected.

Western blot analysis revealed that HIF-1 $\alpha$ was detected in the control cells, but was expressed rarely in the siHIF- $1 \alpha$ transfected cells (Fig. 1A). The results of VEGF ELISA are shown in Fig. 1B. VEGF levels decreased in the supernatants of siHIF-1 $\alpha$-transfected cells compared to levels found in vector-transfected cells. The siHIF-1 transfection led to an $\sim 30 \%$ decrease in the level of VEGF.

The effect of the ERK kinase inhibitor, PD98059, on vector siHIF-1 $\alpha$-, and siHIF-2 $\alpha$-transfected cells under normoxic or hypoxic conditions. We further verified VEGF expression via a HIF-1-independent pathway by treating the vector siHIF-1 $\alpha$ transfected cells with the ERK kinase inhibitor, PD98059, before incubation under normoxic or hypoxic conditions. Phospho-ERK expression was decreased in the PD98059treated cells in each cell line as determined by Western blotting of cell lysates. The down-regulation of ERK expression was clearly demonstrated in the hypoxic condition. HIF-1 expression was also decreased in the PD98059-treated cells grown under hypoxic conditions (Fig. 2A). VEGF expression by ELISA did not differ in the cell lines regardless of PD98059 treatment under hypoxic conditions (Fig. 2B).
A

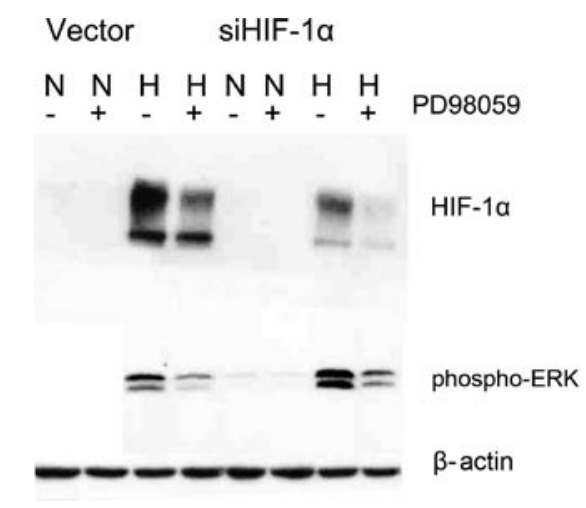

B

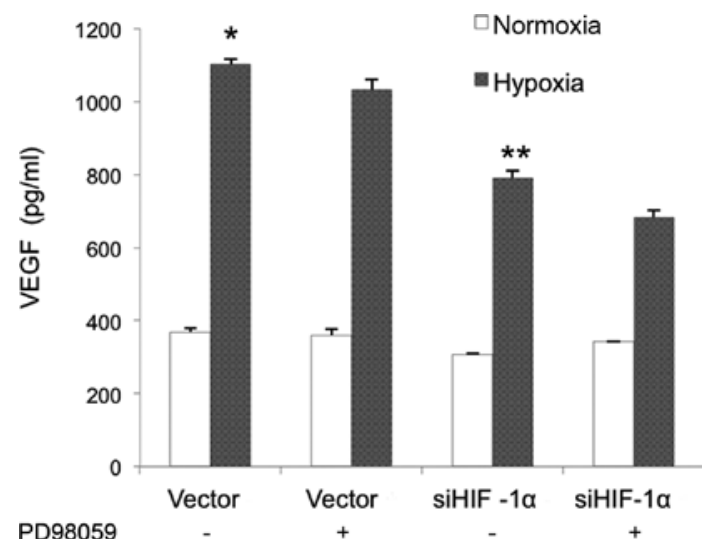

Figure 2. Effect of the ERK kinase inhibitor, PD98059, in vector siHIF-1 $\alpha$ transfected cells. Western blotting revealed that phospho-ERK proteins were expressed under the hypoxic condition without ERK kinase inhibitor treatment. HIF-1 $\alpha$ expression was up-regulated under hypoxic conditions, but was decreased by treatment of cells with the ERK kinase inhibitor (A). Treatment of cells with ERK kinase inhibitor did not affect the expression of VEGF in the vector, siHIF- $1 \alpha$-transfected cells (B). N, normoxia; H, hypoxia; * $\mathrm{p}<0.05$ vs. vector normoxia; ${ }^{* *} \mathrm{p}<0.05$ vs. vector hypoxia.

Effect of the PI3K inhibitor, LY294002, on vector siHIF-1 $\alpha$ transfected cells under normoxic or hypoxic conditions. We treated cells transfected with vector and siHIF-1 $\alpha$ with the PI3K inhibitor, LY294002, before incubation under normoxic or hypoxic conditions. HIF-1 was clearly expressed in all cells incubated under hypoxic conditions. HIF-1 expression was not affected by treatment with LY294002 in vector siHIF-1 $\alpha$ transfected cells (Fig. 3A).

VEGF expression as determined by ELISA was decreased in cells treated with LY294002 compared with untreated cells under hypoxic conditions. VEGF expression in siHIF-1 $\alpha$ transfected cells treated with LY294002 decreased significantly under hypoxic conditions (Fig. 3B).

RT-PCR analysis of VEGF mRNA, demonstrated that the VEGF mRNA expression was remarkably elevated in each of the transfected cell lines (vector, siHIF-1 $\alpha$ ) under hypoxic conditions as compared with cells incubated under normoxic conditions. Among the cells incubated under hypoxic conditions, the expression levels of VEGF mRNA were significantly reduced in the LY294002-treated cells (Fig. 4).

The effect of siSP1 transient transfection on cells transfected with vector siHIF-1 $\alpha$ under normoxic or hypoxic conditions. Transient transfection experiments with siSP1 in stably 
A

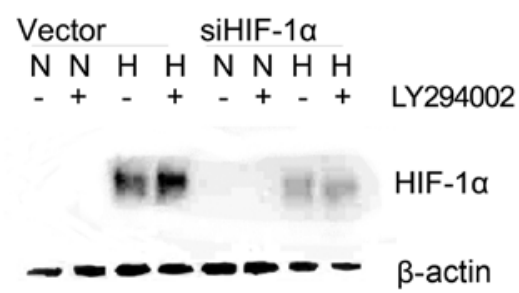

B

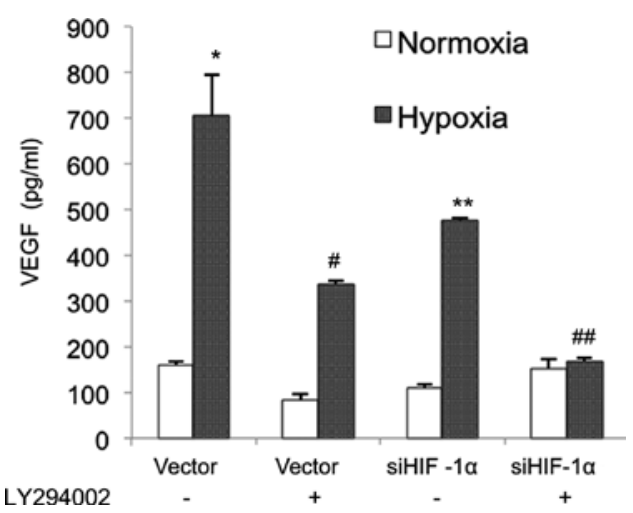

Figure 3. The effect of the PI3K inhibitor, LY294002, in cells transfected with vector siHIF-1 $\alpha$. HIF-1 $\alpha$ expression is elevated under hypoxic condition. There is no difference in HIF-1 $\alpha$ expression between cells treated with the PI3K inhibitor or not treated in any of the transfected cells (A). VEGF expression is decreased in cells treated with the PI3K inhibitor as compared to untreated cells. VEGF expression is decreased by PI3K inhibitor treatment in the siHIF-1 $\alpha$ transfected cells under hypoxia (B). N, normoxia; $\mathrm{H}$, hypoxia; " $\mathrm{p}<0.05$ vs. vector normoxia; ${ }^{* *} \mathrm{p}<0.05$ vs. vector hypoxia; ${ }^{\#} \mathrm{p}<0.05$ vs. vector hypoxia; ${ }^{\# \#} \mathrm{p}<0.05$ vs. siHIF-1 $\alpha$ hypoxia.

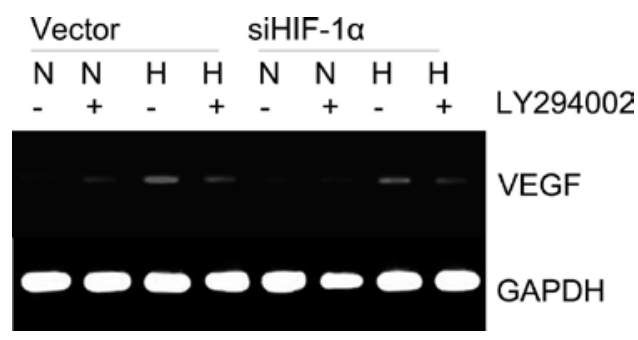

Figure 4. The expression of VEGF is elevated during hypoxia, and VEGF expression is decreased by treatment of cells with the PI3K inhibitor in vector siHIF-1 $\alpha$-transfected cells as compared to untreated cells. N, normoxia; $\mathrm{H}$, hypoxia.

transfected Hep3B cells with vector siHIF-1 $\alpha$, demonstrated that VEGF expression was decreased in the siSP1-transfected cells under hypoxic conditions. VEGF expression in the siHIF-1 $\alpha$-transfected cells was significantly decreased by siSP1 under hypoxic conditions (Fig. 5).

\section{Discussion}

Angiogenesis or neovascularization is a critical step for tumor progression and metastasis. VEGF is a key mediator of tumorassociated angiogenesis $(1,4)$. VEGF is known to be one of the target genes of HIF-1 $\alpha$. HIF-1 plays a central role in the hypoxic expression of various genes. HIF-1 is a heterodimeric basic helix-loop-helix protein that consists of $\alpha$ and $\beta$ subunits (5). HIF-1 $\alpha$ is rapidly degraded in normoxia $(12,13)$, but under

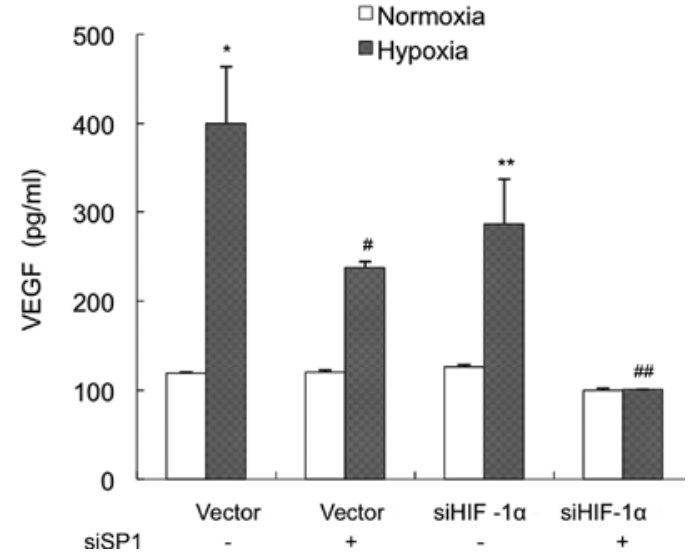

Figure 5. The effect of siSP1 transient transfection in cells stably transfected with vector siHIF-1 $\alpha$. VEGF expression is decreased in cells with siSP1 transient transfection. The expression of VEGF is markedly decreased by siSP1 transient transfection in the siHIF-1 $\alpha$-transfected cells under hypoxia. " $\mathrm{p}<0.05$ vs. vector normoxia; ${ }^{* *} \mathrm{p}<0.05$ vs. vector hypoxia; ${ }^{\#} \mathrm{p}<0.05$ vs. vector hypoxia; ${ }^{\#} \mathrm{p}<0.05$ vs. siHIF-1 $\alpha$ hypoxia.

hypoxic conditions HIF-1 $\alpha$ accumulates, dimerizes with the $\beta$ subunit, translocates to the nucleus, and initiates gene transcription $(14,15)$. It has been reported that HIF-1 induces more than 100 genes under hypoxic conditions (16).

VEGF and the VEGF receptor are up-regulated in HCC $(17,18)$, and overexpression of VEGF is inversely correlated with the prognosis and survival of HCC patients $(19,20)$. VEGF is also one of targets of the anti-cancer drugs sorafenib, sunitinib, and bevacizumab, used to treat HCC (4). HIF-1 is a major transcriptional factor controlling VEGF expression in the malignancy $(21,22)$. A significant positive correlation was found between HIF-1 $\alpha$, VEGF, metastasis, and microvessel density $(23,24)$, while an inverse correlation was shown with tumor differentiation (23). The differences in survival rates among a VEGF-positive group of patients and a VEGFnegative group were significant, whereas the differences in the survival rates among a HIF-1 $\alpha$-negative group and a HIF-1 $\alpha$ positive group were not significant (24).

A more in-depth understanding of the molecular pathogenesis of HCC has resulted in more effective treatment of this malignancy; however, the molecular basis for the induction of VEGF expression in HCC is largely undefined (25). Therefore, the aim of the present study was to investigate the molecular mechanism of VEGF expression, especially its regulation via a HIF-1-independent pathway, in HCC. To increase our understanding of the function of HIF-1 and to identify a HIF-1-independent pathway that controls VEGF expression in hypoxia, loss-of-function studies were performed using an siRNA approach. Though VEGF expression was decreased in the siHIF-1 $\alpha$-transfected cells under hypoxia, significant VEGF expression was still detected. We hypothesized that growth signal pathways were involved in the HIF-1-independent VEGF expression because tumor growth progresses in cancer cells under hypoxia. PD98059 had no effect on VEGF expression while PI3K inhibition and siSP1transient transfection led to decreased VEGF expression. In this study, we found that the main HIF-1-independent pathway regulating VEGF expression in the Hep3B cell line is the Akt/ PI3K pathway, through SP1 (Fig. 6). 


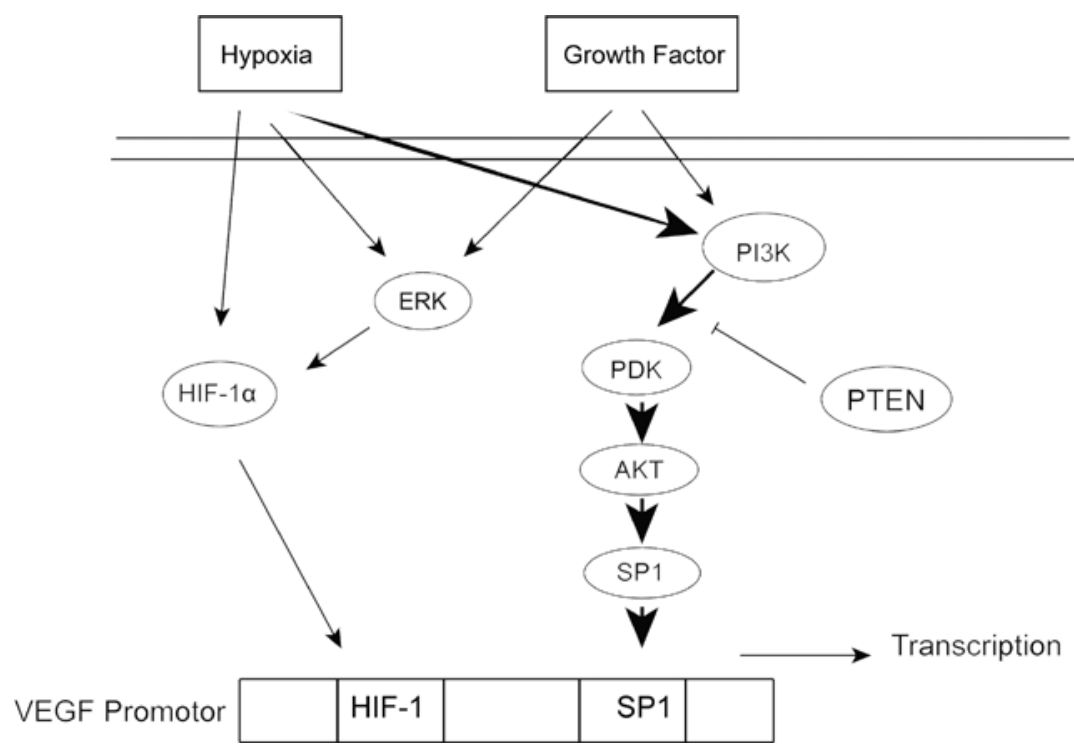

Figure 6. Schematic pathway of the regulation of the VEGF promoter under hypoxia. The dark arrows indicate HIF-1 independent pathways.

There have been additional reports on HIF-1-independent pathways activated during hypoxia. Tumor hypoxia accelerates cancer invasion by up-regulating the expression of ETS-1 and matrix metalloproteinase (MMP) in HCC cell lines (8). Furthermore, cells derived from HIF- $1 \alpha$ knockout embryos demonstrate a significant induction of VEGF in response to hypoxia, suggesting that HIF- $1 \alpha$ may not be the only transcriptional activator of VEGF $(9,10)$. The HIF-1-independent regulation of VEGF may be cell-type specific. It has been reported that a HIF-1-independent regulatory luciferase construct of colon cancer cell lines was unresponsive to hypoxia in the HepG2 and the Panc-1 (pancreatic cancer) cell lines (26). These results indicate that HIF-1 may be the primary mediator of the hypoxic regulation of VEGF in some cell types, but that other cell types can possess various alternative mechanisms.

The PI3K/Akt pathway is commonly activated in human cancer (27). Increased PI3K/Akt activity has been implicated in cell proliferation, adhesion, migration, invasion, and apoptosis (28-30). There are numerous reports showing that PI3K signaling is involved in the regulation of HIF-1 $\alpha$ activity. Growth factors, such as EGF and HER2 (31) and androgens (32), increase HIF-1 $\alpha$ synthesis under normoxic conditions through PI3K/Akt activation. Under hypoxic and normoxic conditions, Akt-activation leads to a decrease in HIF-1 $\alpha$ protein degradation (33). One study suggested that SP1 can augment the HIF-1mediated hypoxic induction of the carbonic anhydrase IX gene (34). However, others have reported that the activity of $\mathrm{PI} 3 \mathrm{~K} / \mathrm{Akt}$ is not necessary for the activation of HIF, nor is it essential for its induction by hypoxia $(35,36)$. Akt contributes to translation of HIF-1 $\alpha$ and can certainly amplify the induction of HIF-1 $\alpha$ under hypoxia in many cell lines although it is not required for HIF-1 $\alpha$ induction under hypoxia (27). In one study, the loss of DNA-binding activity of SP1 proteins was observed in cells incubated under hypoxic conditions (37). In our study, the regulation of VEGF expression by the Akt/ PI3K pathway in the Hep3B cell line does not seem to involve the HIF-1 pathway. SP1 is a key transcription factor in VEGF expression under hypoxia, serving as an alternative to HIF-1. Therefore, the role of the PI3K pathway in VEGF regulation appears to be cell-type specific, and this may be the reason why some studies have questioned its role in the hypoxic response $(14,35,36)$.

In conclusion, we have demonstrated that tumor hypoxia accelerates angiogenesis by up-regulating VEGF expression. There is evidence that HIF-1 is not essential for the hypoxic induction of VEGF in HCC cell lines. VEGF can be induced by hypoxia through HIF-1-dependent and HIF-1-independent mechanisms. Also, the Akt/PI3K activation through SP1 is a critical pathway in VEGF expression under tumor hypoxia by which HIF-1 is not mediated. It will be important to define its clinical role, such as its use as a prognostic factor or as molecular targeted therapy in a large number of tumor samples. SP1, through the PI3K/Akt pathway which is commonly involved in tumorigenesis, is another potent activator of VEGF in a HIF-1-independent manner. This suggests that tumors employ various pathways for angiogenesis under conditions of hypoxia to survive and even to proliferate. Furthermore, these findings indicate the possibility that there may be another therapeutic target in addition to HIF-1 to block angiogenic responses under hypoxia in cancer.

\section{References}

1. Harris AL: Hypoxia - a key regulatory factor in tumour growth. Nat Rev Cancer 2: 38-47, 2002.

2. Hockel M and Vaupel P: Tumor hypoxia: definitions and current clinical, biologic, and molecular aspects. J Natl Cancer Inst 93: 266-276, 2001.

3. Harada H, Kizaka-Kondoh S, Li G, et al: Significance of HIF-1active cells in angiogenesis and radioresistance. Oncogene 26: 7508-7516, 2007.

4. Pang RW and Poon RT: From molecular biology to targeted therapies for hepatocellular carcinoma: the future is now. Oncology 72 (Suppl 1): 30-44, 2007.

5. Ferrara N, Gerber HP and LeCouter J: The biology of VEGF and its receptors. Nat Med 9: 669-676, 2003.

6. Kim KR, Moon HE and Kim KW: Hypoxia-induced angiogenesis in human hepatocellular carcinoma. J Mol Med 80: 703-714, 2002.

7. Gasparini G, Longo R, Toi M and Ferrara N: Angiogenic inhibitors: a new therapeutic strategy in oncology. Nat Clin Pract Oncol 2: 562-577, 2005. 
8. Miyoshi A, Kitajima Y, Ide T, et al: Hypoxia accelerates cancer invasion of hepatoma cells by upregulating MMP expression in an HIF-1 $\alpha$-independent manner. Int J Oncol 29: 1533-1539, 2006.

9. Levy AP, Levy NS and Goldberg MA: Post-transcriptional regulation of vascular endothelial growth factor by hypoxia. J Biol Chem 271: 2746-2753, 1996.

10. Ryan HE, Lo J and Johnson RS: HIF-1 alpha is required for solid tumor formation and embryonic vascularization. EMBO J 17: 3005-3015, 1998.

11. Pore N, Jiang Z, Gupta A, Cerniglia G, Kao GD and Maity A: EGFR tyrosine kinase inhibitors decrease VEGF expression by both hypoxia-inducible factor (HIF)-1-independent and HIF-1dependent mechanisms. Cancer Res 66: 3197-3204, 2006.

12. Cockman ME, Masson N, Mole DR, et al: Hypoxia inducible factor-alpha binding and ubiquitylation by the von Hippel-Lindau tumor suppressor protein. J Biol Chem 275: 25733-25741, 2000.

13. Kamura T, Sato S, Iwai K, Czyzyk-Krzeska M, Conaway RC and Conaway JW: Activation of HIF1 alpha ubiquitination by a reconstituted von Hippel-Lindau (VHL) tumor suppressor complex. Proc Natl Acad Sci USA 97: 10430-10435, 2000.

14. Diaz-Gonzalez JA, Russell J, Rouzaut A, Gil-Bazo I and Montuenga L: Targeting hypoxia and angiogenesis through HIF-1alpha inhibition. Cancer Biol Ther 4: 1055-1062, 2005.

15. Semenza GL: HIF-1: mediator of physiological and pathophysiological responses to hypoxia. J Appl Physiol 88: 1474-1480, 2000

16. Gordan JD and Simon MC: Hypoxia-inducible factors: central regulators of the tumor phenotype. Curr Opin Genet Dev 17: 71-77, 2007.

17. Mise M, Arii S, Higashituji $\mathrm{H}$, et al: Clinical significance of vascular endothelial growth factor and basic fibroblast growth factor gene expression in liver tumor. Hepatology 23: 455-464, 1996.

18. von Marschall Z, Cramer T, Hocker M, Finkenzeller G, Wiedenmann B and Rosewicz S: Dual mechanism of vascular endothelial growth factor upregulation by hypoxia in human hepatocellular carcinoma. Gut 48: 87-96, 2001.

19. Poon RT, Ng IO, Lau C, et al: Serum vascular endothelial growth factor predicts venous invasion in hepatocellular carcinoma: a prospective study. Ann Surg 233: 227-235, 2001.

20. Chao Y, Li CP, Chau GY, et al: Prognostic significance of vascular endothelial growth factor, basic fibroblast growth factor, and angiogenin in patients with resectable hepatocellular carcinoma after surgery. Ann Surg Oncol 10: 355-362, 2003.

21. Maltepe E, Schmidt JV, Baunoch D, Bradfield CA and Simon MC: Abnormal angiogenesis and responses to glucose and oxygen deprivation in mice lacking the protein ARNT. Nature 386: 403-407, 1997.

22. Keith B, Adelman DM and Simon MC: Targeted mutation of the murine arylhydrocarbon receptor nuclear translocator 2 (Arnt2) gene reveals partial redundancy with Arnt. Proc Natl Acad Sci USA 98: 6692-6697, 2001.

23. Ding L, Chen XP and Wang HP: Expression and clinical significance of HIF-1 $\alpha$ protein in hepatocellular carcinoma tissues. Zhonghua Gan Zang Bing Za Zhi 12: 656-659, 2004 (In Chinese).

24. Huang GW, Yang LY and Lu WQ: Expression of hypoxiainducible factor 1alpha and vascular endothelial growth factor in hepatocellular carcinoma: Impact on neovascularization and survival. World J Gastroenterol 11: 1705-1708, 2005.
25. Wagner K, Peters M, Scholz A, et al: Activin A stimulates vascular endothelial growth factor gene transcription in human hepatocellular carcinoma cells. Gastroenterology 126: 1828-1843, 2004.

26. Mizukami Y, Li J, Zhang X, Zimmer MA, Iliopoulos O and Chung DC: Hypoxia-inducible factor-1-independent regulation of vascular endothelial growth factor by hypoxia in colon cancer. Cancer Res 64: 1765-1772, 2004.

27. Pore N, Jiang Z, Shu HK, Bernhard E, Kao GD and Maity A: Akt1 activation can augment hypoxia-inducible factor-1alpha expression by increasing protein translation through a mammalian target of rapamycin-independent pathway. Mol Cancer Res 4: 471-479, 2006.

28. Haas-Kogan D, Shalev N, Wong M, Mills G, Yount G and Stokoe D: Protein kinase B (PKB/Akt) activity is elevated in glioblastoma cells due to mutation of the tumor suppressor PTEN/MMAC. Curr Biol 8: 1195-1198, 1998.

29. Cantley LC and Neel BG: New insights into tumor suppression: PTEN suppresses tumor formation by restraining the phosphoinositide 3-kinase/AKT pathway. Proc Natl Acad Sci USA 96: 4240-4245, 1999.

30. Yamada KM and Araki M: Tumor suppressor PTEN: modulator of cell signaling, growth, migration and apoptosis. J Cell Sci 114: 2375-2382, 2001

31. Laughner E, Taghavi P, Chiles K, Mahon PC and Semenza GL: HER2 (neu) signaling increases the rate of hypoxia-inducible factor lalpha (HIF-1alpha) synthesis: novel mechanism for HIF-1-mediated vascular endothelial growth factor expression. Mol Cell Biol 21: 3995-4004, 2001.

32. Mabjeesh NJ, Willard MT, Frederickson CE, Zhong $\mathrm{H}$ and Simons JW: Androgens stimulate hypoxia-inducible factor 1 activation via autocrine loop of tyrosine kinase receptor/ phosphatidylinositol 3'-kinase/protein kinase B in prostate cancer cells. Clin Cancer Res 9: 2416-2425, 2003.

33. Zhong H, Chiles K, Feldser D, et al: Modulation of hypoxiainducible factor 1alpha expression by the epidermal growth factor/phosphatidylinositol 3-kinase/PTEN/AKT/FRAP pathway in human prostate cancer cells: implications for tumor angiogenesis and therapeutics. Cancer Res 60: 1541-1545, 2000.

34. Kaluz S, Kaluzova M and Stanbridge EJ: Expression of the hypoxia marker carbonic anhydrase IX is critically dependent on SP1 activity. Identification of a novel type of hypoxiaresponsive enhancer. Cancer Res 63: 917-922, 2003.

35. Alvarez-Tejado M, Alfranca A, Aragones J, Vara A, Landazuri MO and del Peso L: Lack of evidence for the involvement of the phosphoinositide 3-kinase/Akt pathway in the activation of hypoxia-inducible factors by low oxygen tension. J Biol Chem 277: 13508-13517, 2002

36. Arsham AM, Plas DR, Thompson CB and Simon MC: Phosphatidylinositol 3-kinase/Akt signaling is neither required for hypoxic stabilization of HIF-1 alpha nor sufficient for HIF-1-dependent target gene transcription. J Biol Chem 277: 15162-15170, 2002.

37. Kang HT, Ju JW, Cho JW and Hwang ES: Down-regulation of Sp1 activity through modulation of O-glycosylation by treatment with a low glucose mimetic, 2-deoxyglucose. J Biol Chem 278: 51223-51231, 2003. 